\title{
Germanene structure enhancement by adjacent insoluble domains of lead
}

\author{
Ting-Yu Chen $\odot,{ }^{1}$ David Mikolas, ${ }^{1}$ Santosh Chiniwar $\odot,{ }^{1}$ Angus Huang, ${ }^{1}$ Chung-Huang Lin, ${ }_{1}^{1}$ Cheng-Maw Cheng $\odot,{ }^{2}$ \\ Chung-Yu Mou $\odot,{ }^{1,3}$ H.-T. Jeng, ${ }^{1,3,{ }^{*}}$ Woei Wu Pai, ${ }^{4,5, \dagger}$ and S.-J. Tang $\odot^{1,2,3, *}$ \\ ${ }^{1}$ Center for Quantum Technology and Department of Physics, National Tsing Hua University, Hsinchu 30013, Taiwan \\ ${ }^{2}$ National Synchrotron Radiation Research Center (NSRRC), Hsinchu 30076, Taiwan \\ ${ }^{3}$ Institute of Physics, Academia Sinica, Taipei 11529, Taiwan \\ ${ }^{4}$ Center for Condensed Matter Sciences, National Taiwan University, Taipei 106, Taiwan \\ ${ }^{5}$ Department of Physics, National Taiwan University, Taipei 106, Taiwan
}

(Received 4 November 2020; revised 2 July 2021; accepted 26 July 2021; published 11 August 2021)

\begin{abstract}
Growth of Xene, referred to as the analog of graphene made of atoms other than carbon, with higher lattice quality and more intrinsic electronic structures, is an important challenge to the development of two-dimensional (2D) materials. We report an approach using $\mathrm{Pb}$-atom deposition to striped-phase (SP) germanene grown on $\mathrm{Ag}(111)$ to trigger it to a quasifreestanding phase (QP). Using low-energy electron diffraction (LEED) and scanning tunneling microscopy (STM), we demonstrate that the 2D monatomic Pb layer, instead of being on top, grows side-by-side with QP germanene, not only driving the SP-to-QP transition but further enhancing the lattice order of QP as revealed by the dramatic evolution of LEED-spot shape and uniform STM-imaged array of moiré patterns. Originally oriented at $30^{\circ}$ to $\operatorname{Ag}(111)$, the QP germanene transits first to a narrow distribution of rotations then to two sharp domains at $\pm 3^{\circ}$ from $30^{\circ}$, while the monoatomic $\mathrm{Pb}$ domains twist from $\pm 4.3^{\circ}$ to $\pm 9.5^{\circ}$ with respect to $\mathrm{Ag}(111)$. The analysis of higher-order coincidence for both layers on $\mathrm{Ag}(111)$ is in complete agreement with LEED results and reveals a picture of two insoluble monatomic layers driving each other to a more optimal configuration. Moreover, the corresponding density of states measured by scanning tunneling spectroscopy for quality-enhanced QP germanene exhibits a clear V-shape distribution at the energy $\sim 0.4 \mathrm{eV}$ above Fermi level, indicating the Fermi-Dirac cone, which agrees with our density functional theory calculation.
\end{abstract}

DOI: 10.1103/PhysRevResearch.3.033138

\section{INTRODUCTION}

Growth of a monatomic layer with a honeycomb lattice has been a hot topic since the discovery of graphene [1], the analogs of which belong to the family of two-dimensional (2D) Xenes [2] such as silicene [3,4], germanene [5-8], stanene [9,10], plumbene [11], borophene [12], bismuthine [13], gallenene [14], phosphorene [15], arsenene [16], antimonene [17], selenene [18], and tellurene [19]. Among them, the first four members belonging to the group 14 elements of the periodic table are considered the first-generation Xenes [20]. Their electric properties such as Dirac-like or spintronic transports were originally revealed from their intrinsic electronic structures of the freestanding forms, which were derived from first-principles calculations. However, from the experimental side, 2D Xenes must be grown on a substrate, which usually hinders the formation of a freestanding Xene due to diffusion through the interfaces [21], large strains

\footnotetext{
*jeng@ @hys.nthu.edu.tw

†wpai@ntu.edu.tw

${ }^{\ddagger}$ sjtang@phys.nthu.edu.tw
}

Published by the American Physical Society under the terms of the Creative Commons Attribution 4.0 International license. Further distribution of this work must maintain attribution to the author(s) and the published article's title, journal citation, and DOI. caused by lattice mismatches [5], or strong electron-electron hybridization between 2D Xenes and the substrates [5,13], leaving the perfect epitaxial growth of $2 \mathrm{D}$ Xenes with exclusively intrinsic electronic structures a tremendous challenge.

It is commonly understood that a perfect epitaxial growth lies in the integral ratio between the lattices of an overlayer and a substrate, the so-called lattice match. Such a condition leads to a regular and periodic correspondence between the atoms of an overlayer and a substrate to optimize the interaction between them and hence reach a stable configuration. When the ratio is nonintegral that it can be expressed as that of two integers without common factors, it is termed as higherorder coincidence (HOC), for which a correspondence still exists, yet with a much larger period than the common multiple of the two integers [22,23]. This is manifested by the moiré patterns, as commonly observed from 2D monatomic layers that have lattice mismatches with underlying substrates [24]. It sometimes occurs that the overlayer lattice spontaneously rotates for a few degrees (both clockwise and counterclockwise) from the original orientation, e.g., $\pm 5^{\circ}$ for $3-\mathrm{ML} \mathrm{Pb}$ layer/Ge(111) [25], $\pm 5.6^{\circ}$ for 1.8-ML Pb layer/Si(111) [26], $\pm 4^{\circ}$ for 1 - ML $\mathrm{Pb}$ layer/ $\mathrm{Ag}(111)$ [24], and $\pm 4^{\circ}$ to $\pm 11^{\circ}$ for various submonolayer $\mathrm{C}_{60}$ phases on $\mathrm{Cu}(111)$ [27], to form a better HOC with the substrate; the density of correspondence increases substantially undergoing such a slight rotation. The rotation angle is thus a balance between the lattice strain and the density of HOC, and the latter could be enhanced via a 


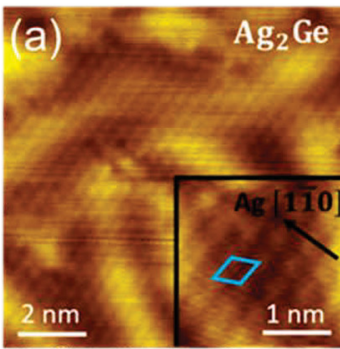

(d)

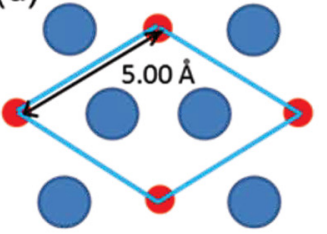

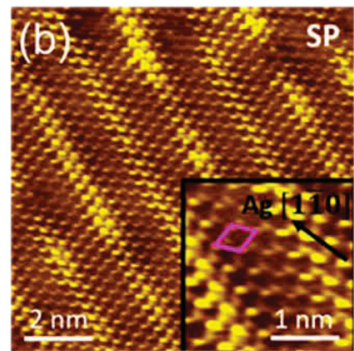

(e)

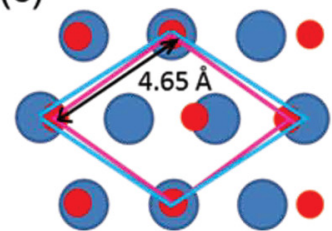

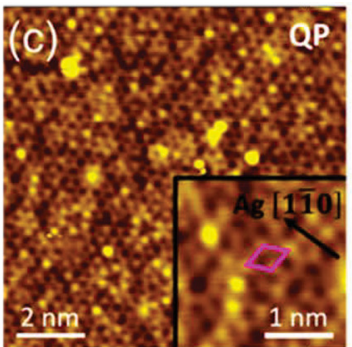

(f)

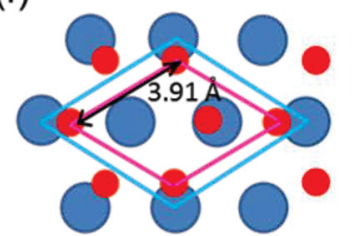

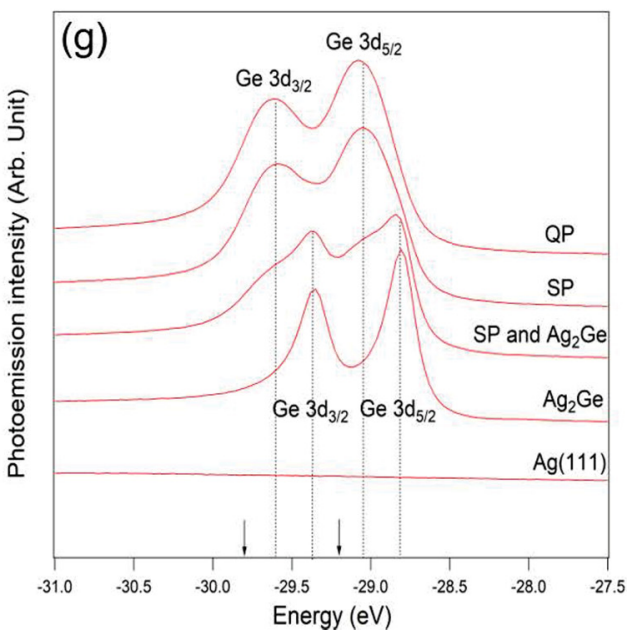

FIG. 1. Formation and evolution of germanene on $\mathrm{Ag}(111)$. (a)-(c) Scanning tunneling microscopy (STM) topographic images for $\mathrm{Ag}_{2} \mathrm{Ge}$ surface alloy phase, striped phase (SP), and quasifreestanding phase [QP; image size, tunneling sample bias, and current: $10 \times 10 \mathrm{~nm}, 0.018 \mathrm{~V}$, and $0.75 \mathrm{nA}$ for (a), $10 \times 10 \mathrm{~nm},-0.04 \mathrm{~V}$, and $0.91 \mathrm{nA}$ for (b), $10 \times 10 \mathrm{~nm}, 0.105 \mathrm{~V}$, and $0.73 \mathrm{nA}$ for (c)]. (d)-(f) The corresponding lattice models along with their unit cells denoted by rhombi and lattice-constant values. Ag and Ge atoms are denoted by blue and red colors. (g) Core-level photoemission spectra of Ge $3 d$ for the three phases and an additional coexisting phase of $\mathrm{Ag}_{2} \mathrm{Ge}$ surface alloy and SP germanene.

changed lattice strain accompanied with an altered rotating angle. Covalent bonding is more accommodating to the lattice strain than metallic bonding so the covalent-bonded Xenes such as germanene and silicene of the first generation can serve as good playgrounds to investigate the interplay between the lattice strain and the density of HOC. It is noteworthy that the covalent-bond character of graphene has brought several interesting surface features such as wrinkles, ripples, and crumples [28], which might also be observed on covalentbonded Xenes.

Our previous study [5] showed that the formation of germanene on $\mathrm{Ag}(111)$ evolved through three stages at different Ge coverage, namely, $\mathrm{Ag}_{2} \mathrm{Ge}$ surface alloy, striped-phase (SP) germanene, and quasifreestanding-phase (QP) germanene. The scanning tunneling microscopy (STM) images that characterize these three phases as well as their lattice models are exhibited in Fig. 1 [5]. As seen from Figs. 1(d)-1(f), the lattices of the three phases are within the frame of $\operatorname{Ag}(111)$ $\sqrt{3} \times \sqrt{3} R 30^{\circ}$, as indicated by the blue rhombus. The unit cells of the SP and QP are marked by pink rhombi. All the rhombi are superimposed onto the lattices of magnified STM images in the insets of Figs. 1(a)-1(c) for a direct correspondence. For the SP, the Ge-Ge bonds undertake large tensile strains $23 \%$ along the stripe direction to match the underlying $\mathrm{Ag}$ atoms of $\mathrm{Ag}(111)-\sqrt{3} \times \sqrt{3} R 30^{\circ}$ unit cell and a $12 \%$ lattice mismatch with the $\mathrm{Ag}$ atoms along the armchair direction [5]. After extra Ge atoms are deposited to the SP, Ge-Ge bonds in both symmetry directions relax to the QP form with $21 \%$ lattice mismatch but only $\sim 3.7 \%$ compressive strain relative to unstrained freestanding germanene [5]. This relaxation-triggering effect by extra $\mathrm{Ge}$ atoms is interesting since it represents a pure mechanical process which might also be induced by atoms of different species to offer advantages. Figure $1(\mathrm{~g})$ shows the photoemission spectra of the Ge $3 d$ core-level state for the three phases; there is an abrupt chemical shift of $\sim 0.3 \mathrm{eV}$, indicating a sharp de-alloying transition from the $\mathrm{Ag}_{2} \mathrm{Ge}$ surface alloy to SP germanene, and as expected, the energy shift of the $3 d$ core-level peak from SP to QP is small $\sim 0.05 \mathrm{eV}$, with an increase in the peak intensity. The slight shift may have to do with the lattice relaxation, and the broader line shape than that of the $\mathrm{Ag}_{2} \mathrm{Ge}$ surface alloy indicates the lattices of the SP and QP are buckled and more disordered than the $\mathrm{Ag}_{2} \mathrm{Ge}$ surface alloy. The arrows at the horizontal axis indicate the energy positions of atomic $\mathrm{Ge} 3 d$ core-level states.

Motivated by a recent investigation of a lateral heterostructure between germanene and stanene [29], in this paper, we made an interesting attempt as follows: complete SP germanene and coexisting SP and QP germanene were prepared on $\mathrm{Ag}(111)$, and then $\mathrm{Pb}$ atoms were deposited, upon an annealing to $150{ }^{\circ} \mathrm{C}$. The $\mathrm{Pb}$ and $\mathrm{Ge}$ atoms are insoluble below $327.7^{\circ} \mathrm{C}$ [30]. We discovered that $\mathrm{Pb}$ atoms did not just trigger the relaxation of the SP to the QP but also enhanced the QP germanene quality on both lattice and electronic sides via shifting the rotation angles of both the QP [from $0^{\circ}$ to $\pm 3^{\circ}$ with respect to $\left.\operatorname{Ag}(111)-\sqrt{3} \times \sqrt{3} R 30^{\circ}\right]$ and the side-by-side $\mathrm{Pb}$ layer [from $\pm 4.3^{\circ}$ to $\pm 9.5^{\circ}$ with respect to $\mathrm{Ag}(111)-1 \times$ $1]$, as confirmed by low-energy electron diffraction (LEED), STM, and scanning tunneling spectroscopy (STS).

\section{EXPERIMENTAL PROCEDURES AND CALCULATION METHODS}

\section{A. Germanene growth and $\mathrm{Pb}$ deposition}

The germanene samples were prepared on a $\mathrm{Ag}(111)$ single crystal, with Ge evaporated from an effusion cell kept at $965^{\circ} \mathrm{C}$ and with the substrate kept at $150{ }^{\circ} \mathrm{C}$. The deposition rate was $\sim 0.01 \mathrm{ML}$ per minute. Here, $1 \mathrm{ML}$ refers to the number of atoms of the top $\operatorname{Ag}(111)$ layer. The silver crystal was cleaned by repeated sputter-anneal cycles, and its cleanness was verified by sharp LEED diffraction spots and by direct STM imaging. Although the range of conditions for 

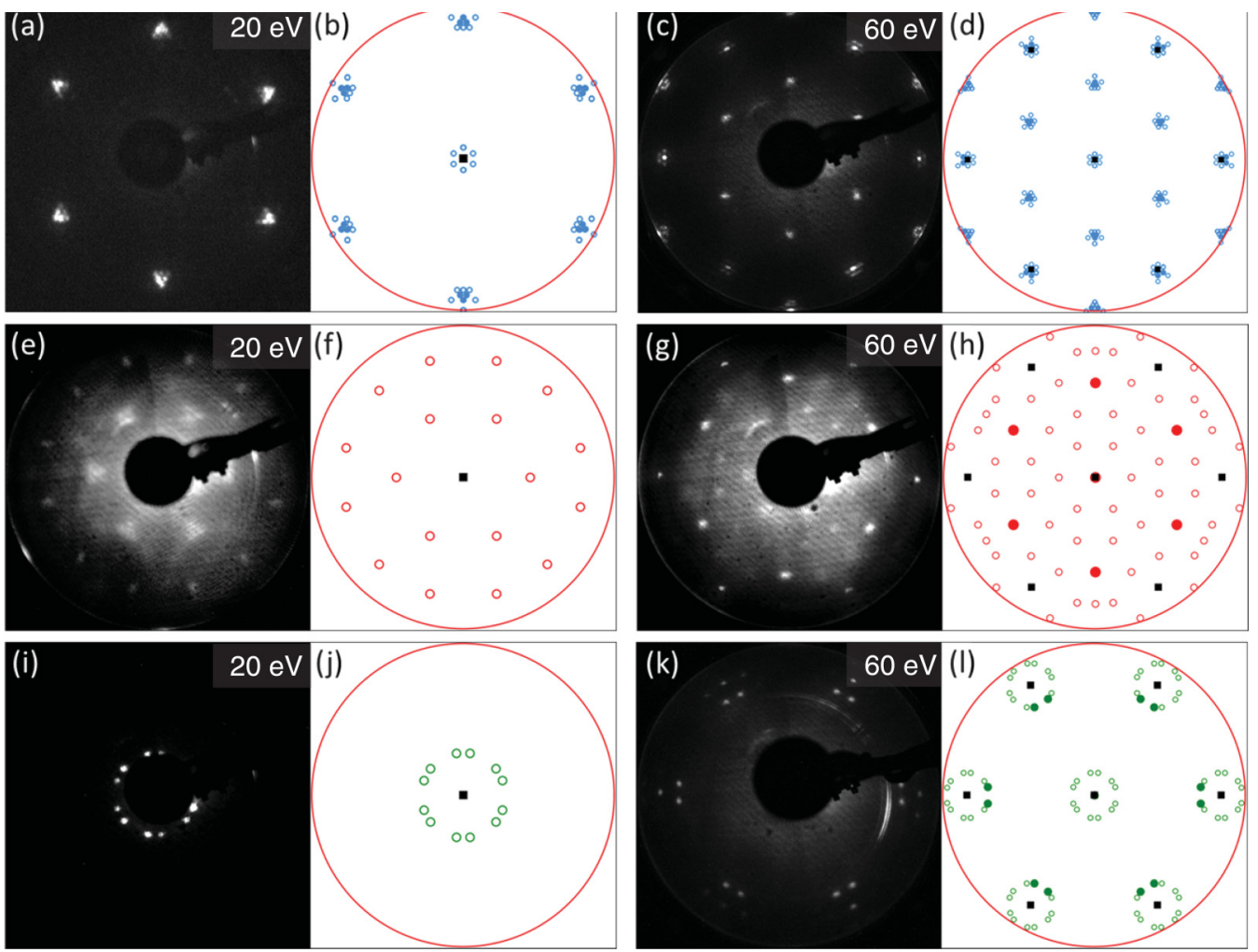

FIG. 2. Low-energy electron diffraction (LEED) patterns and corresponding simulations of (a)-(d) striped phase (SP), (e)-(h) quasifreestanding phase (QP), and (i)-(1) 1-ML $\mathrm{Pb}(111)$ on $\mathrm{Ag}(111)$ at the electron energies of 20 and $60 \mathrm{eV}$ (left and right columns, respectively).

germanene formation has not been explored fully, a slower Ge deposition rate along with a moderately heated substrate facilitates better germanene growth, likely due to reduced random nucleation and enhanced Ge adatom mobility [5]. As for the deposition of $\mathrm{Pb}$, the rate was $0.036 \AA / \mathrm{min}$, as calibrated from the quartz-crystal thickness monitor. The $1.5 \AA$ deposition of $\mathrm{Pb}$ described in the Results and Discussions section refers to the value derived from the deposition rate. Here, 1-ML $\mathrm{Pb}$ layer refers to a monatomic layer with $\mathrm{Pb}(111)$ lattice structure and surface density $\sim 0.68 \mathrm{ML}$ with respect to $\operatorname{Ag}(111)[24,31,32]$.

\section{B. STM measurement}

All STM/STS scans were performed on a commercial omicron LT-STM (RHK SPM-1000 control system) in the Ultra High Vacuum at liquid nitrogen temperature $77 \mathrm{~K}$ and with homemade chemical-etched tungsten tips.

\section{Photoemission measurement}

The core-level photoemission spectra were measured with a Scienta R4000 energy analyzer and with $p$-polarized synchrotron radiation $(80 \mathrm{eV})$ from the undulator beamline 21B1-U9 at the National Synchrotron Radiation Research Center in Taiwan. The energy and angular resolutions were $10 \mathrm{meV}$ and $0.3^{\circ}$, respectively.

\section{LEED simulation}

Throughout all the LEED simulation results shown in this paper, we use lattice constants 2.889 and $3.501 \AA$ [33] of bulk
$\operatorname{Ag}(111)$ and $\mathrm{Pb}(111)$ crystal surfaces and 4.65 and $3.91 \AA$ for SP and QP germanene from previous measurements [5]. The muffin-tin energy was adjusted in each simulation to output similar amounts of diffraction spots as those in measured LEED patterns.

\section{E. DFT calculation}

The first-principles simulations based on density functional theory (DFT) were performed using Vienna Ab initio Simulation Package (VASP) [34-36]. The projector augmented wave pseudopotential [37,38] with Ceperley-Alder type [39] exchange-correlation functional was used in local-density approximation calculations. The germanene lattice constants matching the experiment results were adopted with the ion positions optimized until the residual force was $<0.02 \mathrm{eV} / \AA$.

\section{RESULTS AND DISCUSSIONS}

Figure 2 introduces the LEED patterns of the SP, QP, and 1-ML $\mathrm{Pb}(111)$ layer on $\mathrm{Ag}(111)$ at the electron energies of 20 and $60 \mathrm{eV}$, respectively, along with the simulated counterparts using the lattice models shown in Fig. 1, and the relation for the multiple scattering at the interface, namely, the linear combinations of the reciprocal vectors of the overlayer and the substrate, $\vec{k}=n_{1} \vec{G}_{\text {overlayer }}+n_{2} \vec{G}_{\operatorname{Ag}(111)}\left(n_{1}\right.$ and $n_{2}$ are integers) [5]. Note that, in all the LEED simulation results displayed, the dark, light blue, red, and green colors denote the diffractions from $\operatorname{Ag}(111), \mathrm{SP}, \mathrm{QP}$ germanene, and 1-ML $\mathrm{Pb}$ layer, respectively. Filled and open circles represent the diffraction spots resulting from single and multiple scattering. 


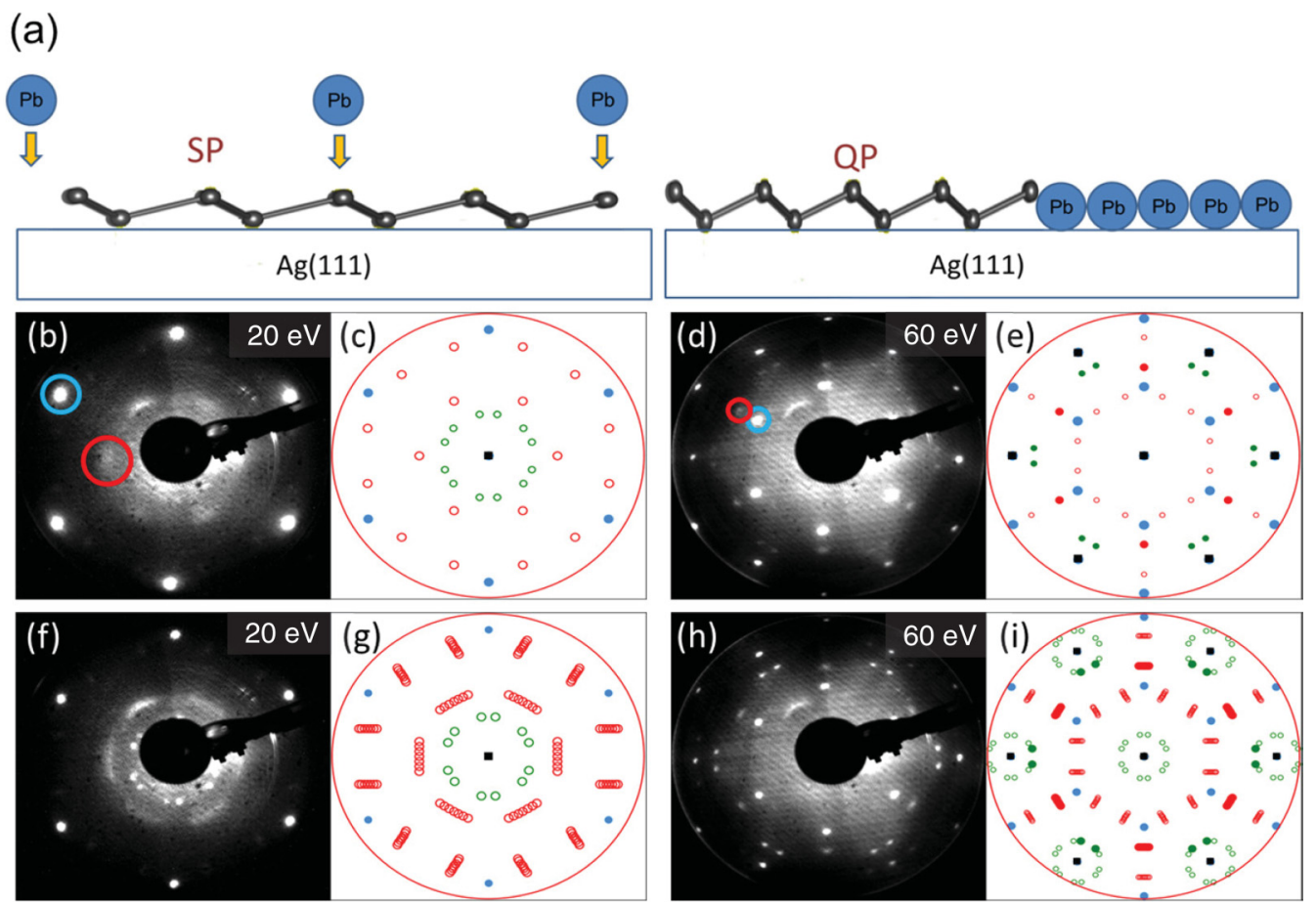

FIG. 3. Pb-atom deposition to striped-phase (SP) germanene. (a) Schematic illustration. (b)-(e) Low-energy electron diffraction (LEED) patterns and corresponding simulations of $1.5-\AA \mathrm{Pb}$ onto SP germanene at $20 \mathrm{eV}$ (left) and $60 \mathrm{eV}$ (right). (f)-(i) LEED patterns and corresponding simulations of further $1.5-\AA \mathrm{Pb}$ deposition at $20 \mathrm{eV}$ (left) and $60 \mathrm{eV}$ (right) with blue and red circles indicating SP and quasifreestanding phase $(\mathrm{QP})$ associated spots, as described in the text.

Lower electron energy enlarges the reciprocal spacing on the LEED screen so that the moiré spots can be observed and resolved more clearly at $20 \mathrm{eV}$. In addition, since moiré spots are derived from multiple scattering of electrons between the overlayer and the substrate, their spot shapes magnify the effects from strains, twisting, and deformation from the interface. In Fig. 2(a), for electron energy of $20 \mathrm{eV}$, the LEED spots centered at the $\left(\frac{1}{3} \frac{1}{3}\right)$ points of the $\operatorname{Ag}(111)-\sqrt{3} \times \sqrt{3} R 30^{\circ}$ structure are surrounded with a triangle array of satellite spots. We have previously reported that these moirélike spots correspond to the long-ranged period of stripes in the armchair direction [5]. The triangle shape is due to the sixfold symmetry of the underlying $\operatorname{Ag}(111)$ causing the three domains of the SP germanene oriented $120^{\circ}$ from each other. At $60 \mathrm{eV}$ [Fig. 2(c)], indeed moirélike spots shrink toward the centered $\left(\frac{1}{3} \frac{1}{3}\right)$ points, and the new satellite LEED spots around $\mathrm{Ag}(111)$ spots at (10) points emerge in the outer region. For QP germanene, at $20 \mathrm{eV}$, there are 12 spots in the outer and six broad spots in the inner region, as shown in Fig. 2(e). According to our simulation result [Fig. 2(f)], the 12 outer spots stem from double scattering of the electrons at the interface, and the six inner broad spots represent moiré around the (00) center point. The broadness, which is not simulated, is ascribed to the actual lattice-disordered nature of QP germanene [5], revealing the threefold interfacial strains from the triangular shape of the moiré spots. Figure 2(i) shows the moiré patterns of a 1-ML $\mathrm{Pb}(111)$ hexagonal layer on top of $\operatorname{Ag}(111)$, which comprises two domains $\pm 4^{\circ}$ relative to $\mathrm{Ag}(111)$ according to the simulation in Fig. 2(j). Figure 2(k) displays the corresponding LEED patterns at 60 eV showing the six single-scattering $\mathrm{Ag}(111)$ spots at (10) points surrounded by moiré spots. They are still simulated very well, as shown in Fig. 2(1).

Figure 3(a) illustrates our approach of using $\mathrm{Pb}$ atoms to trigger relaxation of SP germanene to the QP. Note that the deposition of $\mathrm{Pb}$ is upon an annealing to $150{ }^{\circ} \mathrm{C}$ to ensure side-by-side growth of the $\mathrm{Pb}$ layer with $\mathrm{QP}$ germanene. Figures 3(b) and 3(d) show the LEED patterns of SP germanene after depositing $1.5-\AA \mathrm{Pb}$ atoms onto it at the electron energy of 20 and $60 \mathrm{eV}$, respectively. Compared with the LEED patterns of SP germanene [Figs. 2(a) and 2(c)], one can see the triangular array of SP moirélike spots centered at $\left(\frac{1}{3} \frac{1}{3}\right)$ points evolves to be round, as enclosed by the blue circle, and moreover broad and vague QP moiré spots in the inner region emerge, enclosed by the red circle, in the 20-eV LEED patterns. Correspondingly, single-scattering QP spots, as enclosed by the red circle, emerge in the 60-eV LEED pattern in addition to SP spots centered at $\left(\frac{1}{3} \frac{1}{3}\right)$ points enclosed by blue circles. This demonstrates the triggered relaxation from the SP to the QP [Fig. 3(a)]. Figures 3(c) and 3(e) show the corresponding simulation results that reproduce the LEED data well. Note that the $\mathrm{Ag}_{2} \mathrm{~Pb}$ surface alloy [31,40] can possibly form at this low coverage of $\mathrm{Pb}$ on $\mathrm{Ag}(111)$, which shares the same $\left(\frac{1}{3} \frac{1}{3}\right)$ points in the LEED pattern because of its $\operatorname{Ag}(111)-\sqrt{3} \times \sqrt{3} R 30^{\circ}$ lattice.

Further deposition of additional $1.5-\AA \mathrm{Pb}$ atoms makes the QP moiré spots in the inner region [Fig. 3(f)] more intense, and their broad and diffuse shape sharpens in reciprocal lattice spacing while extending arclike in rotation, indicating the reduction in the uncertainties of the lattice constant with a continuous but limited angular distributions of domains. Comparison with simulation in Fig. 3(g) confirms that the 

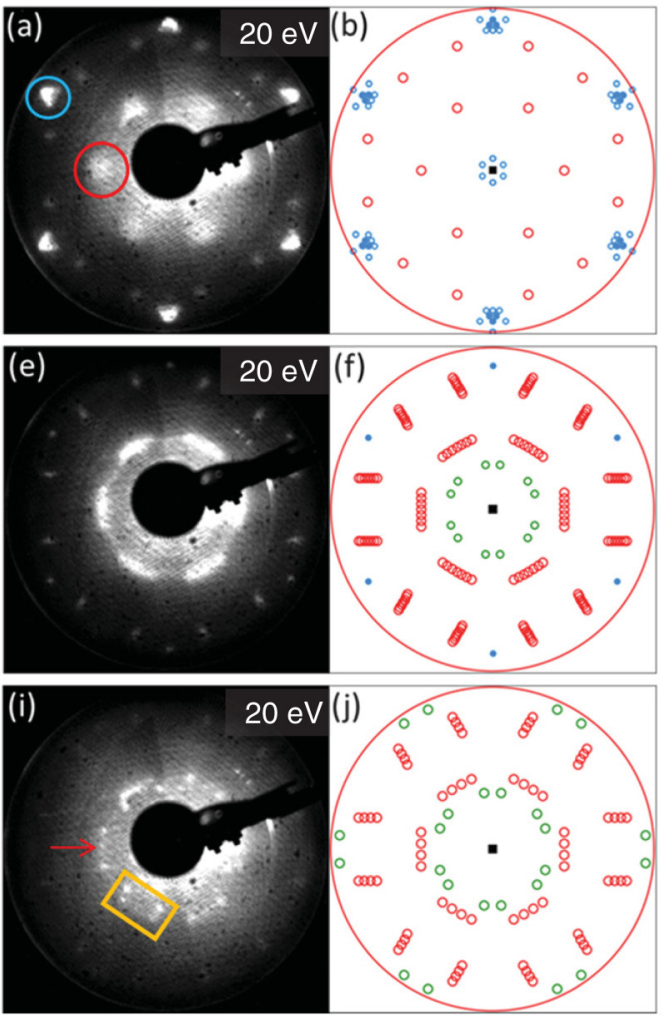
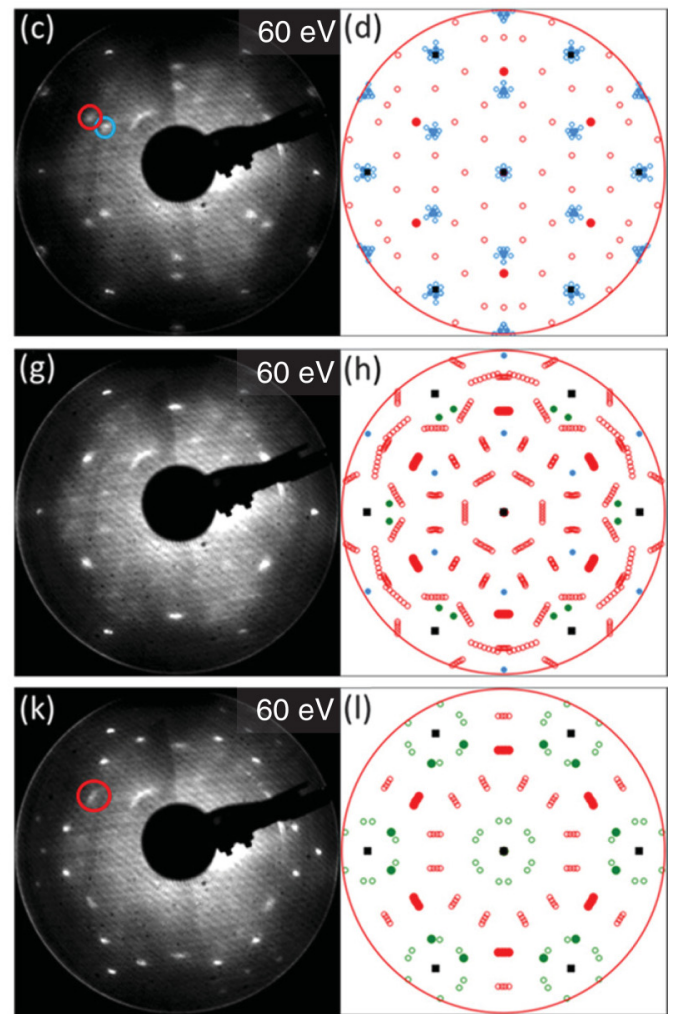

FIG. 4. Pb-atom deposition to coexisting striped-phase (SP) and quasifreestanding-phase (QP) germanene. (a)-(d) Low-energy electron diffraction (LEED) patterns and corresponding simulations of coexisting SP and QP germanene at $20 \mathrm{eV}$ (left) and $60 \mathrm{eV}$ (right). (e)-(h) LEED patterns and corresponding simulations of $1.5-\AA \mathrm{Pb}$ onto coexisting phase at $20 \mathrm{eV}$ (left) and $60 \mathrm{eV}$ (right). (i)-(l) LEED patterns and corresponding simulations of further 1.5- $\mathrm{A} \mathrm{Pb}$ deposition at $20 \mathrm{eV}$ (left) and $60 \mathrm{eV}$ (right).

extent of rotation is within $\pm 3^{\circ}$. The 12 outer QP spots, ascribed to double scattering, more clearly emerge and become elongated for the same reason. In addition, very near the center, six doublets (two of them blocked by the electron gun at the center) show up. These are the moiré spots of the 1-ML $\mathrm{Pb}$ (111) layer comprising domains with $\pm 4^{\circ}$ rotation relative to $\mathrm{Ag}(111)$ as compared with Fig. 2(i). The same moiré spots centered at (10) points of $\mathrm{Ag}(111)$ are also observed in the LEED pattern at $60 \mathrm{eV}$ and are well simulated [Figs. 3(h) and 3(i)]. On the other hand, single-scattering QP spots become more evident and elongated, while the moirélike SP spots further concentrate at the $\left(\frac{1}{3} \frac{1}{3}\right)$ points. It is likely that some residual SP germanene devolves back to the $\mathrm{Ag}_{2} \mathrm{Ge}$ surface alloy phase. Therefore, despite residual SP germanene or $\mathrm{Ag}_{2} \mathrm{Ge}$ surface alloy, the 1-ML $\mathrm{Pb}$ (111) layer starts forming side by side with QP germanene; they appear to mechanically act upon each other, improving the lattice order of QP germanene.

We therefore made a second attempt by depositing $\mathrm{Pb}$ atoms this time to coexisting phases of SP and QP germanene. Their LEED patterns are shown in Figs. 4(a) and 4(c) (20 and $60 \mathrm{eV}$ ). Blue and red circles enclose the moiré spots of the SP and QP in Fig. 4(a) and their corresponding spots (single scattering for the QP) in Fig. 4(c). After an initial 1.5 - $\mathrm{Pb}$-atom deposition upon a $150{ }^{\circ} \mathrm{C}$ annealing, one can see at $20 \mathrm{eV}$ [Fig. 4(e)] that the SP moiré spots centered at $\left(\frac{1}{3} \frac{1}{3}\right)$ points are strongly suppressed, the initially broad QP moiré spots in the inner region have transformed to sharp arcs, and the 12 double-scattering QP spots further elongated. At $60 \mathrm{eV}$ [Fig. 4(g)], the SP spots are negligible, while the singlescattering QP spots intensify, reiterating the main theme of the relaxation of the SP to QP. In addition, there are faint and blurry spots all over regions among the identified spots. They are double-scattering QP spots and $\mathrm{Pb}(111)$ doublets due to the $\pm 4^{\circ}$ rotation relative to $\mathrm{Ag}(111)$, as elucidated from simulation in Fig. 4(h).

This time, the second $1.5-\AA \mathrm{Pb}$ atom deposition produces a dramatic change of the spot shape of QP moirés; the sharp arcs further evolve to a few discrete spots, as shown in Fig. 4(i). All the SP spots vanish, also neglecting the existence of the $\mathrm{Ag}_{2} \mathrm{Ge}$ and $\mathrm{Ag}_{2} \mathrm{~Pb}$ surface alloys since their LEED spots overlap. It is also unlikely that the $\mathrm{Ag}_{2} \mathrm{~Pb}$ surface alloy exists at the interface between $\mathrm{Ag}(111)$ and QP germanene as well as monatomic Pb layer (see Supplemental Material Figs. S3-S5 for more evidence [41]). Upon close examination of the spots enclosed by the yellow rectangle, the most obvious are two pairs of spots. According to our simulation in Fig. 4(j), the outer and inner pairs originate from QP germanene rotating about $\pm 3^{\circ}$ with respect to $\operatorname{Ag}(111)-\sqrt{3} \times \sqrt{3} R 30^{\circ}$ and the $1-\mathrm{ML} \mathrm{Pb}(111)$ layer rotating about $\pm 10^{\circ}$ with respect to $\mathrm{Ag}(111)-1 \times 1$, respectively. The corresponding 60-eV LEED pattern also matches simulation using the same lattice assignment [Figs. 4(k) and 4(1)]. However, the single-scattering spots of QP germanene, as enclosed by the red circle, only exhibit an arclike shape as opposed to the $\pm 3^{\circ}$ doublets due to rotation. We think it is because there are still a few domains 
with smaller rotating angles, as indicated by the red arrow to a blurry spot within the outer pair of the moiré in Fig. 4(i). Therefore, in the simulation [Figs. 4(j) and 4(1)], we incorporate the QP domains of rotation $\pm 1^{\circ}$.

For 2D hexagonal lattices, it is convenient to employ Loeschian numbers $\left(L_{i j}=\sqrt{i^{2}+i j+j^{2}}, i\right.$ and $j=0,1,2,3, \ldots)$ to derive the distance between two arbitrary lattice points. Using an algorithm (see Supplemental Material for details [41]), we searched for all HOC points and computed their densities by solving the relation of $\frac{a_{\text {overlayer }}}{a_{\text {substrate }}}=\frac{\sqrt{L_{i j}}}{\sqrt{L_{l k}}}$ with a corresponding rotation angle between the overlayer and the substrate. Density of HOC relative to overlayer sites is defined as

$$
\rho_{\text {overlayer }}=\frac{1}{k^{2}+k l+l^{2}}, \quad k \text { and } l=0,1,2,3, \ldots,
$$

and rotation angles of coincident domains relative to the substrate are given by

$$
\theta= \pm\left(\arctan \frac{\frac{\sqrt{3}}{2} \boldsymbol{j}}{\boldsymbol{i}+\frac{1}{2} \boldsymbol{j}}-\arctan \frac{\frac{\sqrt{3}}{2} \boldsymbol{l}}{\boldsymbol{k}+\frac{1}{2} \boldsymbol{l}}\right) .
$$

A small variation of the overlayer lattice constant $a_{\text {overlayer }}$ up to $\pm 2 \%$ is allowed to incorporate the strain effect. Note that the strain values are relative to the lattice constant values listed in the Method section.

Figure 5 organizes the analyzed results for the 1-ML $\mathrm{Pb}$ (111) layer on $\mathrm{Ag}(111)$ [Fig. 5(a)] and QP germanene on $\mathrm{Ag}(111)$ [Fig. 5(b)], respectively. Ideally, an overlayer would seek the combination of both the highest HOC density and lowest absolute strain. Figure 5(a) shows clearly that combinations inclined to match this are at $4.3^{\circ}$ and $9.5^{\circ}$. This directly explains why the $1-\mathrm{ML} \mathrm{Pb}(111)$ layer either remains at $\pm 4^{\circ}$ with respect to $\operatorname{Ag}(111)-1 \times 1$ [Figs. 2(j) and 2(1)] or subsequently twists to $\pm 10^{\circ}$ when growing side by side with QP germanene [Figs. 4(j) and 4(1)]. By the same token, one can see from Fig. 5(b) that the most favorable conditions for $\mathrm{QP}$ germanene are at $0^{\circ}$ and $\pm 3.004^{\circ}$ with respect to $\operatorname{Ag}(111)-\sqrt{3} \times \sqrt{3} R 30^{\circ}$, consistent with LEED simulations in Figs. 2(f) and 4(j); the former corresponds to QP germanene alone on $\operatorname{Ag}(111)$, and the latter points to an even higher density of HOC. This highlights the mechanism we exploit; the mechanical interaction between the monatomic $\mathrm{Pb}(111)$ layer and QP germanene twist each other into their most favorable configurations. Note that, at $1.05^{\circ}$, there is a minimum of absolute strain value that can result in minor domains of smaller rotation angles, accounting for blurry QP moiré LEED spots in Fig. 4(i). Based on our HOC analysis, we hereafter denote the QP germanene and 1-ML Pb layer on $\operatorname{Ag}(111)$ as $\sim\left(\frac{7 \sqrt{3}}{9} \times \frac{7 \sqrt{3}}{9}\right) R 30^{\circ}$ and $\left(\sqrt{\frac{28}{19}} \times \sqrt{\frac{28}{19}}\right) R \pm 4.3^{\circ}$ when standing alone, and $\left(\sqrt{\frac{13}{7}} \times \sqrt{\frac{13}{7}}\right) R 30 \pm 3^{\circ}$ and $\left(\sqrt{\frac{19}{13}} \times \sqrt{\frac{19}{13}}\right) R \pm$ $9.5^{\circ}$ when coexisting. Their coverages with respect to the surface density are $1.08,0.679,1.06$, and $0.691 \mathrm{ML}$, respectively. It is important to note that QP germanene standing alone on $\mathrm{Ag}(111)$ is actually disordered with a distribution of strains and domain rotation, as reflected from broad moiré spots in Fig. 2(e), so its notation is only approximate. (a)

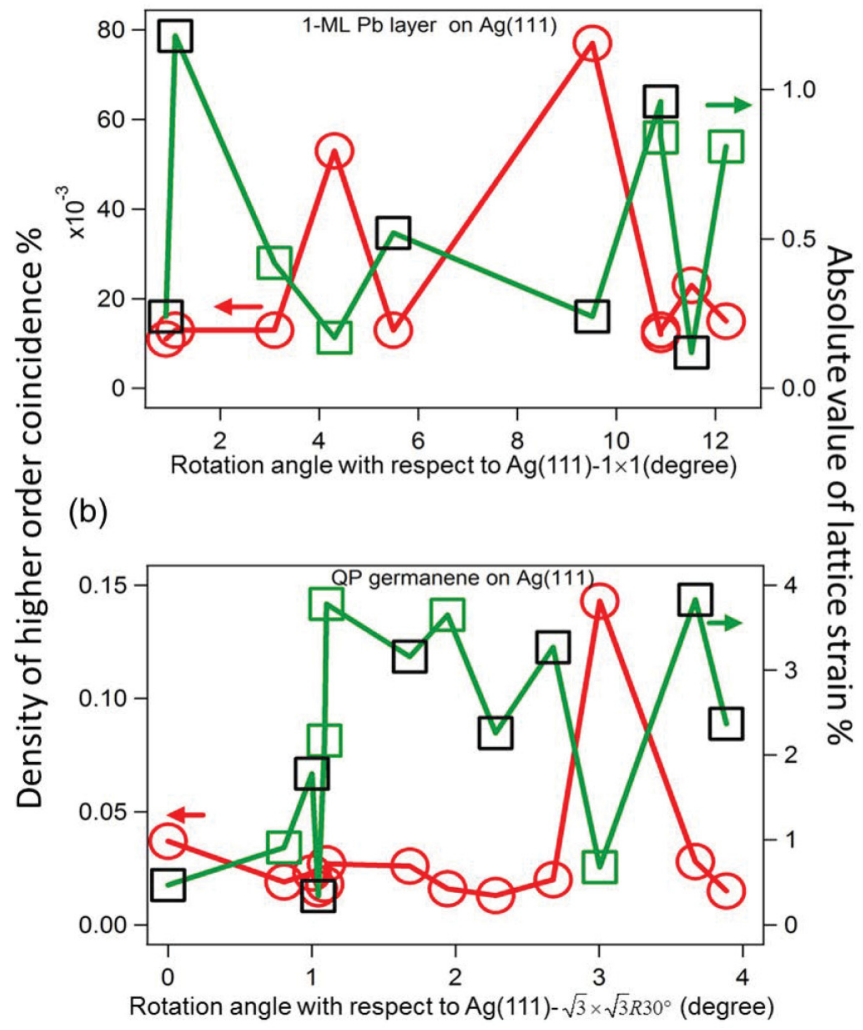

FIG. 5. Summary of higher-order coincidence (HOC) searches for (a) 1-ML $\mathrm{Pb}$ (111) layer and (b) quasifreestanding-phase (QP) germanene on $\operatorname{Ag}(111)$ with density of HOC in red referenced to the left axis and the absolute value of lattice strain, positive in green and negative in dark, referenced to the right axis vs rotation angles with respect to $\operatorname{Ag}(111)-1 \times 1$ and $\operatorname{Ag}(111)-\sqrt{3} \times \sqrt{3} R 30^{\circ}$, respectively.

Corresponding to the case of the LEED patterns in Figs. 4(i) and 4(k), Figs. 6(a) and 6(b) display the STM topographic views of QP germanene coexisting side by side with the monatomic $\mathrm{Pb}(111)$ layer (see Supplemental Material Fig. S6 for more STM topography [41]). Figure 6(a) shows the sharp $\operatorname{Ag}(111)$ step edges separated by $120^{\circ}$, one of which is denoted as the $\mathrm{Ag}$ [1 $1 \overline{10}$ ] close-packed direction. Figure 6(b) is cropped from the lower-left corner of the former, showing the magnified view near the boundary between the monatomic $\mathrm{Pb}(111)$ layer and QP germanene; a line profile of height is superimposed to indicate the smoothness of the former and corrugation of the latter due to the buckled nature. Figure 6(c) shows the magnified view of the monatomic $\mathrm{Pb}$ layer of a single domain. The atoms of the $\mathrm{Pb}(111)$ hexagonal lattice are resolved, along with the $\mathrm{Pb} / \mathrm{Ag}$ moiré period about $1.3 \mathrm{~nm}$, as indicated by one side of the rhombus marking the unit cell of moiré. The corresponding Fourier transform (FT) is shown in Fig. 6(d). The six $\operatorname{Ag}(111)$ spots in the outward region show up due to the transparency of $\mathrm{Pb}$ films [42], serving as reference for the $\mathrm{Pb}$ moiré spots. The white arrow pointing to one $\mathrm{Pb}$ moiré spot, coincident with a lattice spot of the $\mathrm{Pb}(111)$ layer, and the yellow arrow pointing to a $\mathrm{Ag}(01)$ spot are separated by $9^{\circ}$ from each other. The extracted moiré period of $1.3 \mathrm{~nm}$ and the angle of $9^{\circ}$ are close to the $1.26 \mathrm{~nm}$ 

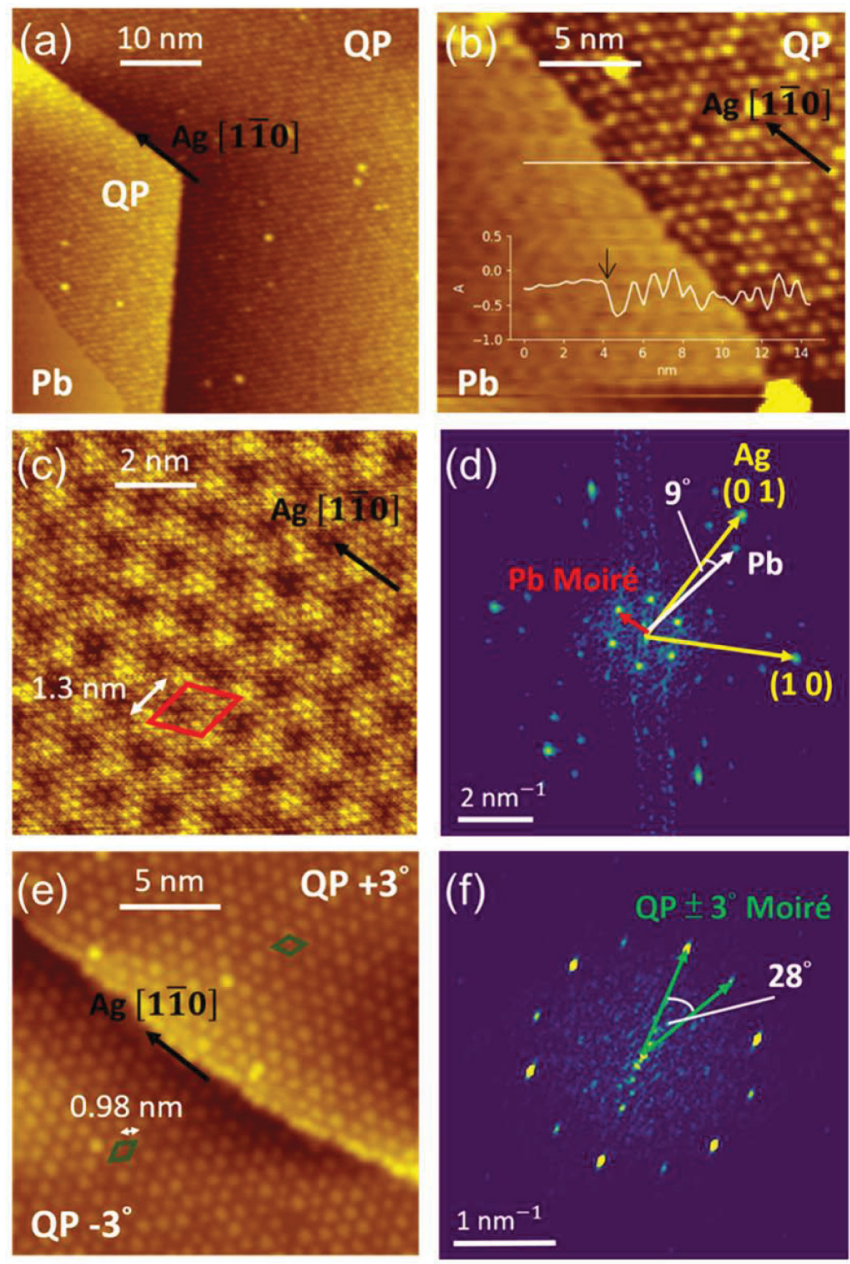

FIG. 6. Scanning tunneling microscopy (STM) images for coexisting quasifreestanding-phase (QP) germanene and monatomic $\mathrm{Pb}$ layer. (a) The topographic view of coexisting $\mathrm{QP}$ germanene domains separated by $\mathrm{Ag}$ step edges and a monatomic $\mathrm{Pb}$ layer. (b) The topographic view near the boundary between monatomic $\mathrm{Pb}$ layer and QP germanene, cropped from the bottom left of (a). The line profile of height is superimposed with an arrow indicating the position of boundary [ $50 \times 50 \mathrm{~nm}, 1.05 \mathrm{~V}$, and $0.47 \mathrm{nA}$ for (a) and $20 \times 20 \mathrm{~nm}, 1.05 \mathrm{~V}$, and $0.47 \mathrm{nA}$ for (b)]. (c) The magnified view of monatomic $\mathrm{Pb}$ layer $(10 \times 10 \mathrm{~nm}, 0.002 \mathrm{~V}$, and $1.0 \mathrm{nA})$. (d) The Fourier transform of (c). (e) The topographic view of the two domains of QP germanene $\left(\sqrt{\frac{13}{7}} \times \sqrt{\frac{13}{7}}\right) R 30 \pm 3^{\circ}$, with a $\operatorname{Ag}(111)$ step edge as the domain boundary $(20 \times 20 \mathrm{~nm}, 1.0 \mathrm{~V}$, and $0.41 \mathrm{nA})$. (f) The Fourier transform of (e).

and $9.5^{\circ}$ of $\left(\sqrt{\frac{19}{13}} \times \sqrt{\frac{19}{13}}\right) R \pm 9.5^{\circ}$ derived from HOC analysis (see Supplemental Material Table 2 [41]). Figure 6(e) shows the two domains of QP germanene $\left(\sqrt{\frac{13}{7}} \times \sqrt{\frac{13}{7}}\right) R 30 \pm 3^{\circ}$ separated by a $\operatorname{Ag}(111)$ step edge. The individual Ge atoms of the honeycomb lattice are not resolved; instead, each observed cluster lines up in a hexagonal pattern with the moiré period of $\sim 0.98 \mathrm{~nm}$, indicated by one side of the rhombus marking the unit cell of moiré. The corresponding FT image [Fig. 6(f)] shows 12 moiré spots contributed from two domains of QP germanene. The extracted period of $0.98 \mathrm{~nm}$ and the angle of $28^{\circ}$ spanned between two arrows in Fig. 6(f) match agreeably
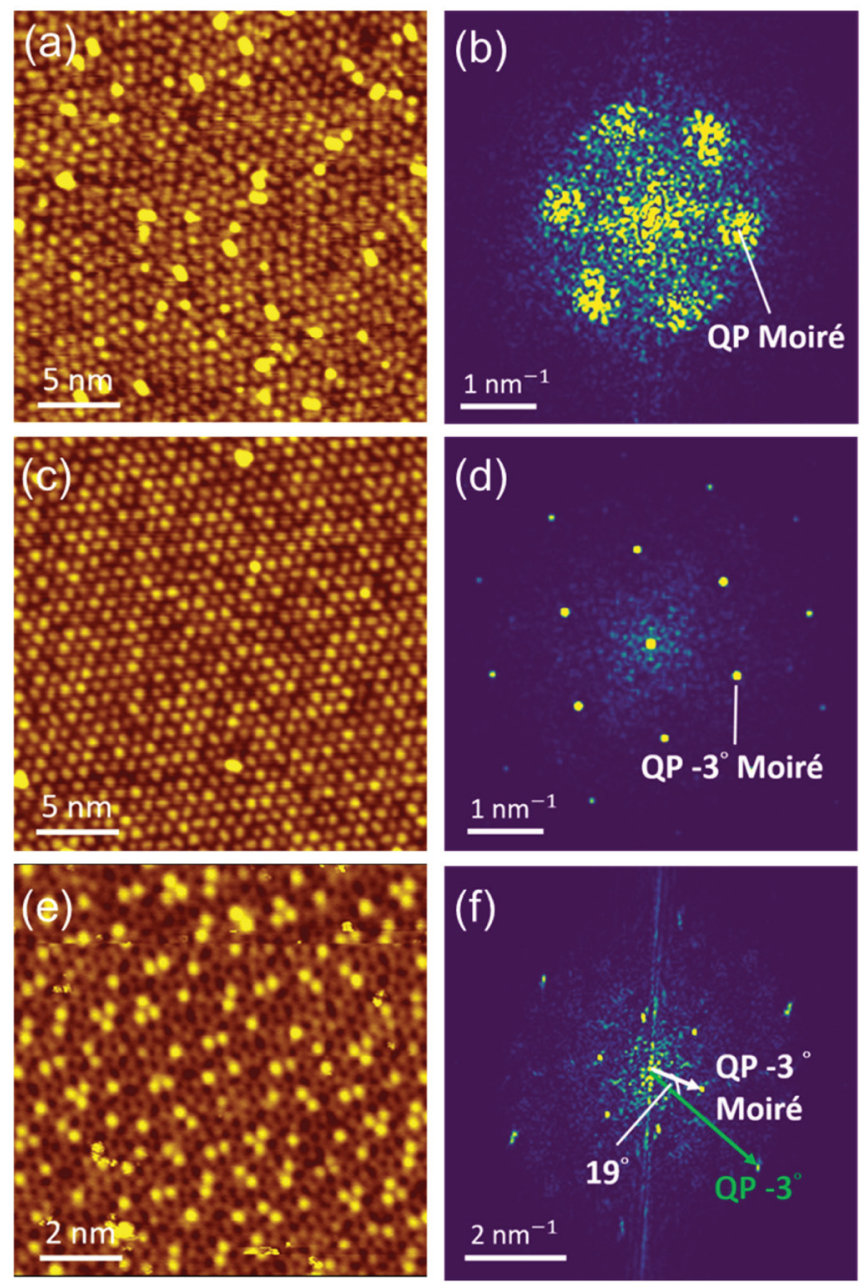

FIG. 7. Comparison between the quasifreestanding-phase (QP) germanene coexisting with monatomic $\mathrm{Pb}$ layer and the standalone QP germanene on $\mathrm{Ag}(111)$ on lattice. (a) Scanning tunneling microscopy (STM) topographic view of moiré lattice of standalone QP germanene on $\operatorname{Ag}(111), \sim\left(\frac{7 \sqrt{3}}{9} \times \frac{7 \sqrt{3}}{9}\right) R 30^{\circ}(25 \times 25 \mathrm{~nm}, 1.0 \mathrm{~V}$, and $1.0 \mathrm{nA}$ ). (b) The Fourier transform of (a). (c) STM topographic view of moiré lattice of QP germanene $\left(\sqrt{\frac{13}{7}} \times \sqrt{\frac{13}{7}}\right) R 30-3^{\circ}$, coexisting with monatomic $\mathrm{Pb}$ layer $(25 \times 25 \mathrm{~nm}, 1.0 \mathrm{~V}$, and $0.45 \mathrm{nA})$. (d) The Fourier transform of (c). (e) The magnified view of QP germanene $\left(\sqrt{\frac{13}{7}} \times \sqrt{\frac{13}{7}}\right) R 30-3^{\circ}$, coexisting with monatomic $\mathrm{Pb}$ layer to reveal honeycomb lattice $(10 \times 10 \mathrm{~nm}, 0.002 \mathrm{~V}$, and $1.36 \mathrm{nA})$. (f) The Fourier transform of (e).

well with the $1.04 \mathrm{~nm}$ and $27.8^{\circ}$ for the moiré structure of $\left(\sqrt{\frac{13}{7}} \times \sqrt{\frac{13}{7}}\right) R 30 \pm 3^{\circ}$ derived from HOC analysis (see Supplemental Material Table 2 [41]).

We make a systematic comparison between the standalone QP germanene and QP germanene $\left(\sqrt{\frac{13}{7}} \times \sqrt{\frac{13}{7}}\right) R 30-3^{\circ}$ coexisting with the monatomic $\mathrm{Pb}$ layer on $\mathrm{Ag}(111)$ in Fig. 7. The lattice order of the latter [Fig. 7(c)] is evidently a lot better than the former [Fig. 7(a)]. The corresponding FT images show in even starker contrast that the six QP moiré spots around the center are much sharper for the latter [Fig. 7(d)], while the former [Fig. 7(b)] shows the same broad moiré spot shapes as those in the LEED pattern 

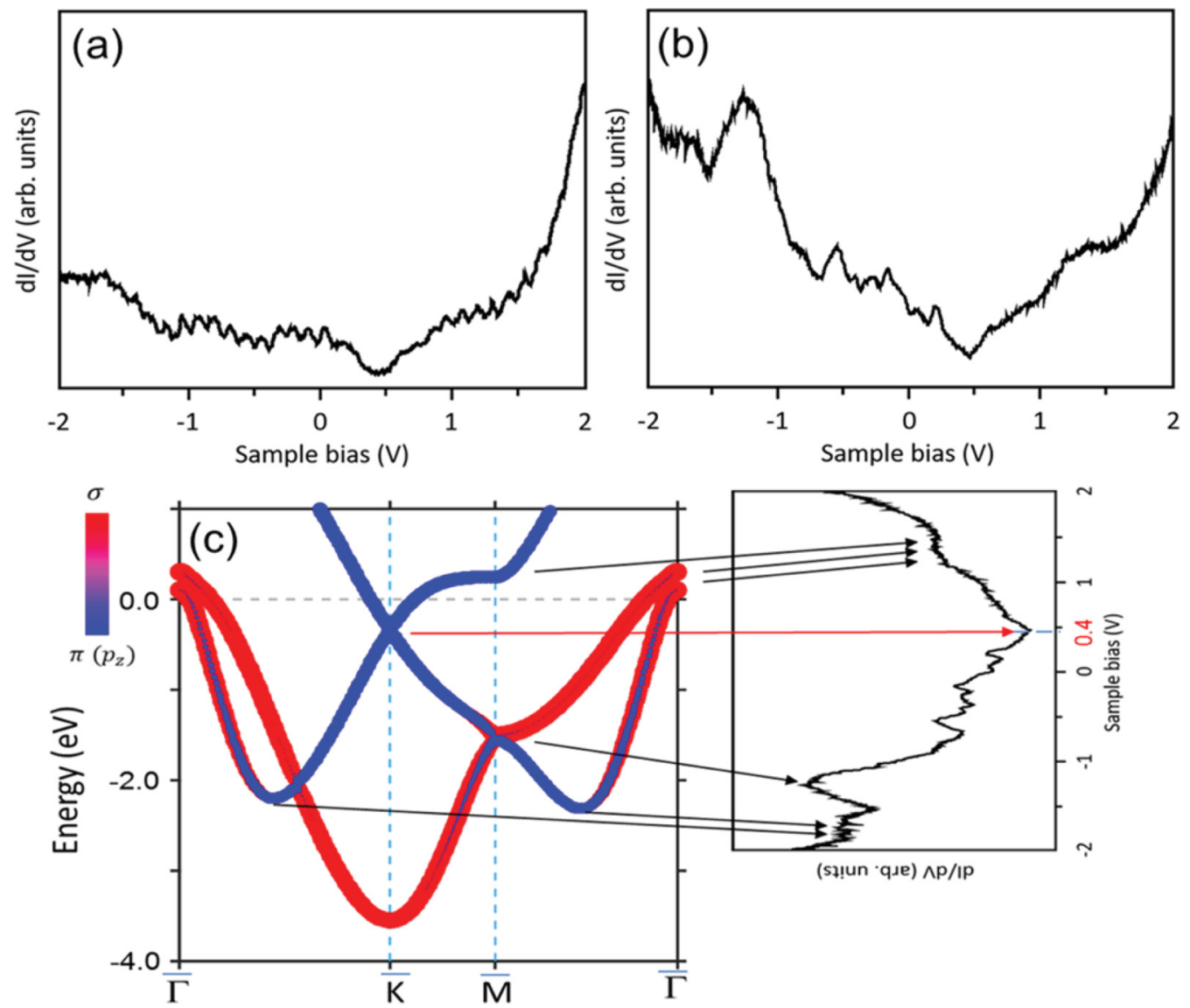

FIG. 8. Comparison between the standalone quasifreestanding-phase (QP) germanene and QP germanene coexisting with monatomic $\mathrm{Pb}$ layer on $\operatorname{Ag}(111)$ on electronic structures. The corresponding scanning tunneling spectroscopy (STS) spectra of (a) standalone QP germanene (tunneling sample bias and current: $0.99 \mathrm{~V}$, and $0.45 \mathrm{nA}$ ) and (b) QP germanene $\left(\sqrt{\frac{13}{7}} \times \sqrt{\frac{13}{7}}\right) R 30-3^{\circ}$, coexisting with monatomic Pb layer (1.0 V and $0.45 \mathrm{nA}$ ) on $\mathrm{Ag}(111)$. (c) The match, as indicated by arrows, between the STS spectrum of the QP germanene coexisting with monatomic $\mathrm{Pb}$ layer in (b) and the energy band structures of a freestanding germanene from DFT calculation. The red arrow indicates the Dirac point. The color-scale bar represents the composite weights of $\sigma$ and $\pi$ bonds for the energy bands.

[Fig. 2(e)]. Figure 7(e) still shows the topography of germanene $\left(\sqrt{\frac{13}{7}} \times \sqrt{\frac{13}{7}}\right) R 30-3^{\circ}$ with a lower sample bias. The image clearly shows a honeycomblike network with slightly raised clusters mostly composed of doublets and triplets integral to the network. Since the clusters are only $\sim 0.4 \AA$ higher according to the extracted height profile (see Supplemental Material Fig. S1 [41]), they are likely the buckled germanene atoms, the configurations of which may have to do with the composite units of honeycombs, such as hexagons, pentagons, etc. Those other than hexagons are likely caused by the defects or interfacial strains [5]. It is also noteworthy that the extracted height profile through the centers of honeycombs is atomically flat, ruling out the possibility of intercalated $\mathrm{Pb}$ atoms in them; however, when viewed at a large scale, there are very few brighter spots with more height $\sim 1.2 \AA$ at random locations (see Supplemental Material Figs. S2(a) and S2(b) [41]), likely inferring adatoms such as $\mathrm{Pb}$ atoms on top of the QP germanene.

Figure 7(f) shows the corresponding FT image in a larger scale, which in addition to the same six moiré spots in the inner region shows six lattice spots in the outer region. The rotation angle between the lattice and the moiré spots is $19^{\circ}$, consistent with $19.1^{\circ}$ between the orientations of the honeycomb lattice and moiré structure for $\left(\sqrt{\frac{13}{7}} \times \sqrt{\frac{13}{7}}\right) R 30-3^{\circ}$ derived from HOC analysis (see Supplemental Material Table 2 [41]).

The STS results [Figs. 8(a) and 8(b)] further show that $\mathrm{QP}$ germanene coexisting with the monatomic $\mathrm{Pb}$ layer on $\mathrm{Ag}(111)$ unravels more evident peak features with enhanced sharpness, which are carefully compared with the energy band structures of a freestanding germanene from DFT calculation [Fig. 8(c)]. While the center of the V-shaped valley at $\sim 0.4 \mathrm{eV}$ in the STS spectrum likely corresponds to the Dirac point, such an assignment needs to be corroborated by other factors. The pronounced peak at $-1.3 \mathrm{eV}$ can be considered as one such indicator because it matches the double saddle points at $\bar{M}$, where a $\pi$ and a $\sigma$ band intersect with each other at also about $-1.3 \mathrm{eV}$. Therefore, the pronounced peak can get smeared out easily once the lattice is disordered enough to deviate the two saddle points from merging, which is likely the case in Fig. 8(a). The small bump, ranging -2.0 to $-1.8 \mathrm{eV}$, beside the pronounced peak corresponds to the bottom of the $\pi$ bands within $\bar{\Gamma}-\bar{M}$ and $\bar{\Gamma}-\bar{K}$. As indicated by other arrows on the top, the small shoulder ranging $1.2-1.6 \mathrm{eV}$ 
corresponds to the saddle point of the unoccupied $\pi$ band at $\bar{M}$ and the top of the $\sigma$ bands at $\bar{\Gamma}$.

\section{CONCLUSIONS}

We report the discovery of an interesting recipe for enhancement of epitaxially grown monatomic layers on a lattice-mismatched substrate using chemical pressure between an insoluble monatomic layer and an existing Xene, in this case 1-ML $\mathrm{Pb}(111)$ and $\mathrm{QP}$ germanene on $\mathrm{Ag}(111)$. The idea is to increase the coverage (surface density) of germanene by occupying the underlying $\operatorname{Ag}(111)$ area with the $\mathrm{Pb}$ layer. Our systematic LEED measurements and detailed simulations indicate that $\mathrm{Pb}$ atoms indeed trigger relaxation of SP germanene to denser QP germanene, and moreover, the interaction between the side-by-side QP germanene and monatomic $\mathrm{Pb}(111)$ layer causes their further rotation from approximately $\mathrm{Ge} / \mathrm{Ag}(111)-\left(\frac{7 \sqrt{3}}{9} \times \frac{7 \sqrt{3}}{9}\right) R 30^{\circ}$ and $\mathrm{Pb} / \mathrm{Ag}(111)-\left(\sqrt{\frac{28}{19}} \times \sqrt{\frac{28}{19}}\right) R \pm 4.3^{\circ}$ to $\mathrm{Ge} / \mathrm{Ag}(111)-$ $\left(\sqrt{\frac{13}{7}} \times \sqrt{\frac{13}{7}}\right) R 30 \pm 3^{\circ}$ and $\mathrm{Pb} / \operatorname{Ag}(111)-\left(\sqrt{\frac{19}{13}} \times \sqrt{\frac{19}{13}}\right) R \pm$ $9.5^{\circ}$, respectively. HOC density analysis for both monatomic layers on $\operatorname{Ag}(111)$ shows that the further rotation of both converts them to more favorable configurations that also produce improved lattice order and more intrinsic electronic structures. Indeed, our LEED, STM, and STS measurements exclusively confirm this. Our results provide an avenue to the goal of utilizing germanene for scientific and industrial purposes by demonstrating a way to enhance the germanene lattice as well as the intrinsic electronic structures.

\section{ACKNOWLEDGMENTS}

This research was supported by the Ministry of Science and Technology of Taiwan (Grant No. MOST 108-2112-M-007021-MY3 for S.-J.T, No. MOST 104-2112-M-002-013-MY3 for W.W.P., and No. MOST 103-2112-M-007-018-MY3 for H.-T.J.). H.-T.J. acknowledges National Center for High-performance Computing, Computer and Information Networking Center-National Taiwan University, Academia Sinica-Innovative Materials and Analysis Technology Exploration, and National Center for Theoretical Sciences, Taiwan, for technical support.
[1] K. S. Novoselov, A. K. Geim, S. V. Morozov, D. Jiang, Y. Zhang, S. V. Dubonos, I. V. Grigorieva, and A. A. Firsov, Electric field effect in atomically thin carbon films, Science 306, 666 (2004).

[2] A. Molle, J. Goldberger, M. Houssa, Y. Xu, S. C. Zhang, and D. Akinwande, Buckled two-dimensional Xene sheets, Nat. Mater. 16, 163 (2017).

[3] P. Vogt, P. De Padova, C. Quaresima, J. Avila, E. Frantzeskakis, M. C. Asensio, A. Resta, B. Ealet, and G. Le Lay, Silicene: Compelling Experimental Evidence for Graphenelike TwoDimensional Silicon, Phys. Rev. Lett. 108, 155501 (2012).

[4] A. Molle, C. Grazianetti, L. Tao, D. Taneja, Md. H. Alam, and D. Akinwande, Silicene, silicene derivatives, and their device applications, Chem. Soc. Rev. 47, 6370 (2018).

[5] C.-H. Lin, A. Huang, W. W. Pai, W.-C. Chen, T.-Y. Chen, T.-R. Chang, R. Yukawa, C.-M. Cheng, C.-Y. Mou, I. Matsuda, T.-C. Chiang, H.-T. Jeng, and S.-J. Tang, Single-layer dual germanene phases on Ag(111), Phys. Rev. Mater. 2, 024003 (2018).

[6] J. Zhuang, C. Liu, Z. Zhou, G. Casillas, H. Feng, X. Xu, J. Wang, W. Hao, X. Wang, S. X. Dou, Z. Hu, and Y. Du, Dirac signature in germanene on semiconducting substrate, Adv. Sci. 5, 1800207 (2018).

[7] J. Yuhara, H. Shimazu, K. Ito, A. Ohta, M. Araidai, M. Kurosawa, M. Nakatake, and G. Le Lay, Germanene epitaxial growth by segregation through $\mathrm{Ag}(111)$ thin films on $\mathrm{Ge}(111)$, ACS Nano 12, 11632 (2018).

[8] M. E. Dávila, L. Xian, S. Cahangirov, A. Rubio, and G. Le Lay, Germanene: a novel two-dimensional germanium allotrope akin to graphene and silicene, New. J. Phys. 16, 095002 (2014).

[9] J. Yuhara, Y. Fujii, K. Nishino, N. Isobe, M. Nakatake, L. Xian, A. Rubio, and G. Le Lay, Large area planar stanene epitaxially grown on $\mathrm{Ag}(111)$, 2D Mater. 5, 025002 (2018).

[10] F.-F. Zhu, W.-J. Chen, Y. Xu, C.-L. Gao, D.-D. Guan, C.-H. Liu, D. Qian, S.-C. Zhang, and J.-F. Jia, Epitaxial growth of two-dimensional stanene, Nat. Mater. 14, 1020 (2015).

[11] J. Yuhara, B. He, N. Matsunami, M. Nakatake, and G. Le Lay, Graphene's latest cousin: plumbene epitaxial growth on a "Nano WaterCube," Adv. Mater. 31, 1901017 (2019).

[12] B. Feng, O. Sugino, R.-Y. Liu, J. Zhang, R. Yukawa, M. Kawamura, T. Iimori, H. Kim, Y. Hasegawa, H. Li, L. Chen, K. Wu, H. Kumigashira, F. Komori, T.-C. Chiang, S. Meng, and I. Matsuda, Dirac Fermions in Borophene, Phys. Rev. Lett. 118 096401 (2017).

[13] F. Reis, G. Li, L. Dudy, M. Bauernfeind, S. Glass, W. Hanke, R. Thomale, J. Schäfer, and R. Claessen, Bismuthene on a SiC substrate: a candidate for a high-temperature quantum spin Hall material view, Science 357, 287 (2017).

[14] V. Kochat, A. Samanta, Y. Zhang, S. Bhowmick, P. Manimunda, S. A. S. Asif, A. S. Stender, R. Vajtai, A. K. Singh, C. S. Tiwary, and P. M. Ajayan, Atomically thin gallium layers from solidmelt exfoliation, Sci. Adv. 4, e1701373 (2018).

[15] L. Li, Y. Yu, G. J. Ye, Q. Ge, X. Ou, H. Wu, D. Feng, X. H. Chen, and Y. Zhang, Black phosphorus field-effect transistors, Nat. Nanotechnol. 9, 372 (2014).

[16] S. M. Beladi-Mousavi, A. M. Pourrahimi, Z. Sofer, and M. Pumera, Atomically thin 2D-arsenene by liquid-phased exfoliation: toward selective vapor sensing, Adv. Funct. Mater. 29, 1807004 (2018).

[17] X. Wu, Y. Shao, H. Liu, Z. Feng, Y.-L. Wang, J.-T. Sun, C. Liu, J.-O. Wang, Z.-L. Liu, S.-Y. Zhu, Y.-Q. Wang, S.-X. Du, Y.-G. Shi, K. Ibrahim, and H.-J. Gao, Epitaxial growth and airstability of monolayer antimonene on $\mathrm{PdTe}_{2}, \mathrm{Adv}$. Mater. 29, 1605407 (2017).

[18] J. Qin, G. Qiu, J. Jian, H. Zhou, L. Yang, A. Charnas, D. Y. Zemlyanov, C.-Y. Xu, X. Xu, W. Wu, H. Wang, and P. D. Ye, Controlled growth of a large-size $2 \mathrm{D}$ selenium nanosheet and 
its electronic and optoelectronic applications, ACS Nano 11, 10222 (2017).

[19] Q. Wang, M. Safdar, K. Xu, M. Mirza, Z. Wang, and J. He, Van der Waals epitaxy and photoresponse of hexagonal tellurium nanoplates on flexible mica sheets, ACS Nano 8, 7497 (2014).

[20] C. Grazianetti, C. Martella, and A. Molle, The Xenes generations: a taxonomy of epitaxial single-element 2D materials, Phys. Status Solidi RRL 14, 1900439 (2020).

[21] R. Bernard, Y. Borensztein, H. Cruguel, M. Lazzeri, and G. Prévot, Growth mechanism of silicene on $\mathrm{Ag}(111)$ determined by scanning tunneling microscopy measurements and ab initio calculations, Phys. Rev. B 92, 045415 (2015).

[22] A. Trampert, O. Brandt, and K. H. Ploog, Crystal Structure of Group III Nitrides (Academic Press, Cambridge, MA, 1998), Vol. 50, Chap. 7.

[23] S.-J. Tang and I. Matsuda, Monatomic Two-Dimensional Layers: Modern Experimental Approaches for Structure, Properties, and Industrial Use (Micro and Nano Technologies), 1st ed. (Elsevier, Amsterdam, 2018), Chap. 3.

[24] S. Chiniwar, A. Huang, T.-Y. Chen, C.-H. Lin, C.-R. Hsing, W.-C. Chen, C.-M. Cheng, H.-T. Jeng, C. M. Wei, W. W. Pai, and S.-J. Tang, Substrate-mediated umklapp scattering at the incommensurate interface of a monatomic alloy layer, Phys. Rev. B 99, 155408 (2019).

[25] S.-J. Tang, C.-Y. Lee, C.-C. Huang, T.-R. Chang, C.-M. Cheng, K.-D. Tsuei, H.-T. Jeng, V. Yeh, and T.-C. Chiang, Electronic versus Lattice Match for Metal-Semiconductor Epitaxial Growth: $\mathrm{Pb}$ on Ge(111), Phys. Rev. Lett. 107, 066802 (2011).

[26] M. Yakes and M. C. Tringides, Probing the buried $\mathrm{Pb} / \mathrm{Si}(111)$ interface with SPA LEED and STM on $\mathrm{Si}(111)-\mathrm{Pb} \alpha \sqrt{3} \times \sqrt{3}$, J. Phys. Chem. A 115, 7096 (2011).

[27] W. W. Pai, C.-L. Hsu, M. C. Lin, K. C. Lin, and T. B. Tang, Structural relaxation of adlayers in the presence of adsorbateinduced reconstruction: $\mathrm{C}_{60} / \mathrm{Cu}(111)$, Phys. Rev. B 69, 125405 (2004).

[28] S. Deng and V. Berry, Wrinkled, rippled and crumpled graphene: an overview of formation mechanism, electronic properties, and applications, Mater. Today 19, 197 (2016).
[29] T. Ogikubo, H. Shimazu, Y. Fujii, K. Ito, A. Ohta, M. Araidai, M. Kurosawa, G. Le Lay, J. Yuhara, Continuous growth and germanene and stanene lateral heterostructures, Adv. Mater. Interfaces 7, 1902132 (2020).

[30] R. W. Olesinski and G. J. Abbaschian, The Ge-Pb (germaniumlead) system, Bull. Alloy Phase Diagr. 5, 374 (1984).

[31] J. Dalmas, H. Oughaddou, G. Le Lay, B. Aufray, G. Tréglia, C. Girardeaux, J. Bernardini, J. Fujii, and G. Panaccione, Photoelectron spectroscopy study of $\mathrm{Pb} / \mathrm{Ag}(111)$ in the submonolayer range, Surf. Sci. 600, 1227 (2006).

[32] J. Yuhara and T. Ako, Two-dimensional Pb-Sn alloy monolayer films on $\mathrm{Ag}(111)$, Appl. Surf. Sci. 351, 83 (2015).

[33] W. Davey, Precision measurements of the lattice constants of twelve common metals, Phys. Rev. 25, 753 (1925).

[34] G. Kresse and J. Hafner, Ab initio molecular dynamics for openshell transition metals, Phys. Rev. B 48, 13115 (1993).

[35] G. Kresse and J. Furthmüller, Efficiency of ab-initio total energy calculations for metals and semiconductors using a plane-wave basis set, Comput. Mater. Sci. 6, 15 (1996).

[36] G. Kresse and J. Furthmüller, Efficient iterative schemes for $a b$ initio total-energy calculations using a plane-wave basis set, Phys. Rev. B 54, 11169 (1996).

[37] G. Kresse and D. Joubert, From ultrasoft pseudopotentials to the projector augmented-wave method, Phys. Rev. B 59, 1758 (1999).

[38] P. E. Blöchl, Projector augmented-wave method, Phys. Rev. B 50, 17953 (1994).

[39] D. M. Ceperley and B. J. Alder, Ground State of the Electron Gas by a Stochastic Method, Phys. Rev. Lett. 45, 566 (1980).

[40] J. Dalmas, H. Oughaddou, C. Léandri, J.-M. Gay, G. Le Lay, G. Tréglia, B. Aufray, O. Bunk, and R. L. Johnson, Ordered surface alloy formation of immiscible metals: the case of $\mathrm{Pb}$ deposited on Ag(111), Phys. Rev. B 72, 155424 (2005).

[41] See Supplemental Material at http://link.aps.org/supplemental/ 10.1103/PhysRevResearch.3.033138 for extra information of experimental data as well as the details of theoretical approach and LEED simulation.

[42] I. B. Altfeder, D. M. Chen, and K. A. Matveev, Imaging Buried Interfacial Lattices with Quantized Electrons, Phys. Rev. Lett. 80, 4895 (1998). 